\title{
CARACTERIZACION DE LA FRACCION INSAPONIFICABLE Y ESTABILIDAD DEL ACEITE DE TRES PALM ERAS DEL GENERO Attalea
}

\author{
Víctor SOTERO ${ }^{1}$, Claudia MERINO $^{1}$, Ericka DAVILA $^{1}$, Kember MEJIA $^{1}$, Jorge VELA $^{1}$, Dora GARCIA $^{2}$ \\ 1 Laboratorio de Sustancias Naturales Bioactivas. Programa PIBA. Instituto de Investigaciones de la Amazonía Peruana. Av. \\ Abelardo Quiñónez. Km 2.5 - Iquitos - Perú. .e-mail: vsotero@ iiap.org.pe \\ 2. Departamento de Química. Facultad de Ingeniería Química. Universidad Nacional de la Amazonía Peruana. Freyre 616 - Iquitos - \\ Perú.e-mail:doegato@ hotmail.com
}

\section{RESUMEN}

En el presente trabajo se determinó la estabilidad del aceite y caracterización de la fracción insaponificable del aceite de tres palmeras del género Attalea: Attalea moorei (AM), Attalea salazariii (AS) y una aun no identificada denominada Attalea sp. (ASP), colectadas en la amazonía peruana entre los años 2009 - 2010. La concentración de aceite en las semillas fue de $23.02 \% .18 .03 \%$ y $19.47 \%$ en peso seco para AM. ASP y AS respectivamente. Se determinó la concentración de $\alpha$ y $\beta$-carotenos y $\alpha$-tocoferol mediante cromatografía de HPLC. La caracterización de la fracción insaponificable se realizó por cromatografía gaseosa. Para evaluar la estabilidad de los aceites estos fueron sometidos a diferentes temperaturas determinándose el índice de peróxidos como indicador de dicha cualidad. La concentración de carotenos fue de 924.32, 795.22 y 2025.99 ug g $^{-1}$ para AS, AM y ASP respectivamente y de $\alpha$-tocoferol fue de 7.5, 7.7 y $15.4 \mathrm{mg} \mathrm{Kg}^{-1}$. La cuantificación total de esteroles fue de 379.0 409.0 y $917.0 \mathrm{mg} \mathrm{Kg}^{-1}$ de aceite en AM, AS y ASP respectivamente, la de hidrocarburos totales de 550 a $731 \mathrm{mg} \mathrm{Kg}^{-}$ ${ }^{1}$ de aceite de parafinas exógenos de $516-682 \mathrm{mg} \mathrm{Kg}^{-1}$ de aceite, siendo la mayor concentración de $\beta$-sitosterol en sus fracciones esterolicas fueron de 54.2, 57.8 y $50.6 \%$ en AM, AS y ASP respectivamente. Los aceites de las tres especies presentan una buena resistencia a la oxidación cuando calentadas hasta $175^{\circ} \mathrm{C}$.

PALABRAS CLAVE: Attalea, aceite, fracción insaponificable, estabilidad.

\section{CHARACTERIZATION OF UNSAPONIFIABEL FRACTION AND STABILITY OF THREE PALMS OIL OF Attalea GENUS}

\begin{abstract}
It was realized the thermal stability and chemical characterization of the unsaponifiable fraction of three oil palm Attalea genus: Attalea moorei (AM), Attalea salazarii (AS) and another even not identifying and called Attalea sp (ASP)) collected from the Peruvian Amazon at 2009 - 2010. The oil concentration at every bean was: 23.02\%, $18.03 \%$ and 19.47 for AM, ASP y AS respectively. The concentration of $\alpha$ and $\beta$ - carotenes and $\alpha$ - tocopherols were determined by means of the chromatography of HPLC. The characterization of the fraction unsaponifiable was carried out for gas chromatography. To evaluate the stability of the oils they were submitted to different temperatures being determined the index of peroxides as indicator of said quality. The concentration of carotenes was of 924,32, 795,22 and 2025,99 ug g-1 for AS, AM and ASP respectively and of was of 7,5, 7,7 and 15,4 mg $\mathrm{Kg}^{-1}$. The total quantification of sterols was of $379,0409,0$ and $917,0 \mathrm{mg} \mathrm{Kg}-1$ of oil in AM, AS and ASP respectively, that of total hydrocarbons from 550 to $731 \mathrm{mg} \mathrm{Kg}-1$ of oil of exogenous paraffins of 516-682 mg Kg-1 of oil, being the greater concentration of $\beta$-sitosterol in their sterolic fractions were of 54.2, 57,8 and 50,6\% in AM, AS and ASP respectively. The oils of the three species present a good resistance to the oxidation when heated until $175^{\circ} \mathrm{C}$
\end{abstract}

KEYW ORDS: Attalea, oil, unsaponifiable fraction, stability 


\section{INTRODUCCIÓN}

El género Attalea esta conformados por palmeras propias del neotrópico, que se encuentran distribuidas desde México hasta Bolivia. Este genero se caracteriza por presentar una identificación muy complicada debido a la sobreposición de caracteres morfológicos (Pintaud, 2008). En la región amazónica se las encuentra sobre todo en rodales naturales, aunque también están presentes en parcelas de productores como resultado de una tala selectiva. En estas localidades estas palmeras son conocidas con los nombres comunes de "shapaja" o "shebón". Sus frutos varían en forma de acuerdo a la especie, pero por lo general son ovoides y presentan de tres a cuatro semillas, ubicadas en forma longitudinal a este. La población rural utiliza las hojas de estas palmeras para la construcción de techos para sus casas y sus frutos para el consumo humano y de animales domésticos (Ferreira, 2005).

Estas palmeras poseen un alto contenido de aceite en sus semillas. Siendo que las especies Attalea moorei (AM). Attalea sp (ASP) y Attalea salazarii (AS), presentan una alta concentración de ácidos grasos saturados como ácido láurico, que varia de 44.4 a $51.8 \%$ y de ácido mirística en el rango de 15.40 18.73\% (Dávila et al., 2011). En general la fracción insaponificable de los aceites ha cobrado bastante interés debido a que contienen sustancias muy importantes como carotenos, tocoferoles y esteroles, los cuales participan en la prevención o tratamiento de ciertas patologías (Chasquibol et al., 2003). Los carotenos son los precursores de la vitamina A, siendo que el más importante por su actividad fisiológica es el $\beta$-caroteno. La vitamina $\mathrm{A}$ además de su función antioxidante es un componente de los pigmentos visuales e interviene en forma esencial en el fenómeno de la visión (Martínez, 2003; Rodríguez-Amaya, 1999). Los tocoferoles son antioxidante naturales de gran actividad y actúan evitando la oxidación de los aceites, y como vitamina $\mathrm{E}$ en el organismo retardan el deterioro celular (Nolasco et al., 2004; Sayago et al., 2007; Fables et al., 2002). Además se ha demostrado que los esteroles (campesterol, estigmasterol, clerosterol, $\beta$-sitosterol, sitostanol, avenasterol) tienen habilidad para bloquear la absorción del colesterol, ciertas investigaciones han revelado que los fitoesteroles bloquean el desarrollo de tumores en el colon, glándulas mamarias y próstata (Chasquibol et al., 2003).

En este sentido el presente trabajo tiene como objetivo contribuir al conocimiento de tres especies del genero Attalea a través de la caracterización química de la fracción insaponificable y estabilidad térmica del aceite de estas especies

\section{MATERIALY MÉTODOS}

\section{MATERIALBIOLÓGICO}

Las especies seleccionadas fueron Attalea moorei (AM). Attalea salazarii (AS) y Attalea sp (ASP) esta última no identificada taxonómicamente y que se encontraba en abundancia en la zona de muestreo. Todas las muestras fueron georeferenciadas (Tabla 1). Las colectas de frutos de AM y ASP, se realizaron en los tres primeros trimestres del 2009 y de AS, se realizó en el tercer trimestre del 2010.

\section{PARTEEXPERIM ENTAL}

El aceite fue extraído por el método de Soxhlet (AOAC, 2005). Para lo cual se somete a un tratamiento térmico según Arrubia et al. (2008). El aceite extraído fue dividido en 5 envases de color ámbar, se sometió el contenido durante dos horas a temperaturas de 125 , 150,175 y $200{ }^{\circ} \mathrm{C}$, mediante baño maría en aceite mineral por dos horas, dejando una de los envases fuera del proceso con el fin de comparar las propiedades del aceite sometido a calentamiento con las del aceite sin calentar para evaluar su estabilidad, mediante el cálculo del índice de peróxido

\section{DETERM INACIONES ANALITICAS}

Se determinó el coeficiente de extinción especifico de los aceites por espectrofotometría en un rango de 190 1100 nm, según Paz et al. (2000). Para los análisis cuantitativos de la fracción insaponificable, se determinaron los hidrocarburos alifáticos, esteroles y alcoholes alifáticos por cromatografía gaseosa, según lo indicado por el reglamento CCE 258-91 (2003). Para el análisis previo de la fracción esterolica, se utilizó la cromatografía de capa fina, utilizando como estándar estigmasterol, según Tano-Debrah \& Ohta (1996). El índice de peróxido se realizó según el método descrito por la AOAC (2005).

\section{RESULTADOS Y DISCUSIÓN}

El rendimiento del aceite en los frutos de las especies del genero Attalea analizados vario de 7.0 a $12.56 \%$ para AM. ASP respectivamente (Tabla 1). Datos muy similares fueron encontrados por Villachica (1996), para los frutos de la palmera babasu (Orbgynia phalerata). Es característica de las especies del género Attalea presentar baja concentración de aceite en relación al peso del fruto, a diferencia de otras especies como Elaeis guineensis (30 a 70\%), Bactris gasipaes H.B.K. (8.3 a 23\%), Jessenia bataua (14 a $22 \%$ ) (Trevejo, 2003).

La concentración de $\beta$-caroteno en el aceite es de 707.55. 3047.98 y 758.10 ug g ${ }^{-1}$ para AM. ASP y AS y 
de a-tocoferol es de 7.7. 7.5 y $15.4 \mathrm{mg} \mathrm{L}^{-1}$ en AM. ASP y AS respectivamente (Tabla 3), comparando con el aceite de aguaje (Mauritia flexuosa), este aceite es superior en la concentración de $\beta$-carotenos ya que Vásquez et al. (2010) trabajando con tres morfotipos reporta para estos un rango de 264.60 - $324.42 \mathrm{ug} \mathrm{g}^{-1}$., aunque muy similar al que se reporta para pijuayo (Bactris gasipaes H.B.K) que proporciona de $1.1 \mathrm{a}$ $22.3 \mathrm{mg} / 100 \mathrm{~g}$ de pulpa (Jatunov et al., 2010) ya que las semillas de Attalea varían de 14.76 a $54.95 \mathrm{mg} / 100 \mathrm{~g}$. La concentración de tocoferoles en aceite es inferior a los que indica Vásquez et al. (2010) para el aguaje con 677.58 a $685.8 \mathrm{mg} \mathrm{L}^{-1}$, aunque superiores al del umarí (Paraqueiba sericea TUL) que según Trevejo (2003) presenta de 16.0 a $17.0 \mathrm{mg} / 100 \mathrm{~g}$ de tocoferoles en el aceite.

El análisis cualitativo para verificar la presencia de estigmasterol se presenta en la Figura 1, donde la placa de cromatografía fina indica la presencia de este compuesto en el aceite de las tres especies.

La concentración de hidrocarburos alifáticos, parafinas exógenos, esteroles y alcoholes alifáticos en la fracción insaponificable de los aceites de las tres especies se muestran en la Tabla 4. Se observa una alta concentración de hidrocarburos totales $(609.0,560.0$ y $731 \mathrm{mg} \mathrm{Kg}^{-1}$ para AM; ASP y AS) y parafinas exógenos (550.0, 516.0 y $682.0 \mathrm{mg} \mathrm{Kg}^{-1}$ para AM; ASP y AS); apreciable cantidad de esteroles (379.0, 917.0 y 409.0 mg Kg ${ }^{-1}$ para AM; ASP y AS) y baja concentración de alcoholes alifáticos $\left(27.0,12.0\right.$ y $13.0 \mathrm{mg} \mathrm{Kg}^{-1}$ para AM;ASPyAS).

Desde el punto de vista nutricional y de salud humana las sustancias más interesantes en esta fracción, son las pertenecientes a la fracción esterólica y, entre estos el estigmasterol (con 17.8. 19.2 y $26.1 \mathrm{mg} \mathrm{Kg}^{-1}$ de aceite para AM; ASP y AS) y, ß-sitosterol (54.2, 57.8 y 50.6 $\mathrm{mg} \mathrm{Kg} \mathrm{K}^{-1}$ para AM. ASP y AS). Comparando las concentración de estigmasterol y $\beta$-sitosterol de las
Attaleas con el aguaje, (Mauritia flexuosa), castaña (Berthellotia excelsa) y umarí (Paraqueiba sericea) (Gómez da Silva et al., 1997; Trevejo, 2003), resultan ser superiores solo en el contenido de estigmasterol y algo menores en cuanto a las concentraciones de $\beta$ sitosterol. Estas sustancias están asociadas a la prevención y tratamiento de ciertos tipos de cáncer. Se ha estudiado al estigmasterol en ratones como un posible antitumoral contra el carcinoma Ehrilch Ascites (Ghosh et al., 2011). El sitosterol, está siendo estudiado como una sustancia que previene el cáncer de próstata (Von Holtz et al., 1998; Awad et al., 2001).

Al graficar los espectros de absorción de los aceites de las semillas en estudio a $25{ }^{\circ} \mathrm{C}$, presentados en la Figura 2, y que se correlacionan con la Tabla 5, donde se presentan los coeficientes de extinción de los aceites comparados con el de oliva (Paz Antolin et al., 2000). Esta información permite determinar la pureza, la adulteración o el enranciamiento del aceite de acuerdo a los parámetros del Reglamento de la Comunidad Económica Europea (CEE) $\mathrm{N}^{\circ}$ 2568/91(1991), de la Comisión del 11 de julio de 1991 se puede considerar que estos aceites, se encuentran en los límites permisibles y se les puede calificar, como aceites de buena calidad, ya que esta norma indica que deben de estar entre 0.1 y 0.8 . Comparados estos con los aceites de oliva, son superiores; esto se podría explicar debido a que se trabajó con aceites crudos y conservaban la presencia de sus antioxidantes naturales (carotenos y tocoferoles). Con los resultados obtenidos de las curvas de la cinética de oxidación de los aceites, presentados en la Figura 2, se observa que estos son bastante estables hasta los $175^{\circ} \mathrm{C}$, y que se oxidan a temperaturas superiores, en cambio el aceite de soja comercial presente mejor estabilidad, esto se puede explicar debido a la adición de antioxidantes sintéticos a este último.

Tabla 1. Georeferencia de Attalea moorei, Attalea sp. y Attalea salazarii.

\begin{tabular}{llcc}
\hline \multirow{2}{*}{ ESPECIE } & \multicolumn{2}{c}{ LUGAR DE COLECTA } & \multicolumn{2}{c}{ GEOREFERENCIAS } \\
\cline { 3 - 4 } & & LATITUD & LONGITUD \\
\hline Attalea salazarii & Carretera lquitos-Nauta Km 67.8 - Maynas - Loreto & 9523032 & 0663807 \\
Attalea sp. & Tamshiyacu - M aynas - Loreto & 9539276 & 0726976 \\
Attalea sp. & Tamshiyacu - Maynas - Loreto & 9546528 & 0721812 \\
Attalea moorei & Tamshiyacu - M aynas - Loreto & 9558762 & 0703603 \\
\hline
\end{tabular}


Tabla 2. Rendimiento de aceite en los frutos de Attalea moorei, Attalea sp. y Attalea salazarii.

\begin{tabular}{lccccccc}
\hline ESPECIE & $\begin{array}{c}\text { PESO } \\
\text { FRUTO, } \\
\mathbf{g}\end{array}$ & $\begin{array}{c}\% \\
\text { ACEITE }\end{array}$ & $\begin{array}{c}(\mathbf{g}) \\
\text { ACEITE/ } \\
\text { SEMILLAS }\end{array}$ & $\begin{array}{c}(\mathbf{g}) \\
\text { ACEITE/ } \\
\text { FRUTO }\end{array}$ & $\begin{array}{c}\% \\
\text { ACEITE/ } \\
\text { FRUTOS }\end{array}$ & $\begin{array}{c}\text { (g) } \\
\text { ACEITE/ } \\
\text { RACIM0 }\end{array}$ & $\begin{array}{c}\text { (g) } \\
\text { ACEITE/ } \\
\text { PLANTA }\end{array}$ \\
\hline A. moorei & 170,26 & 23,02 & 21,61 & 11,91 & 7,00 & 2382,2 & 9528,9 \\
A. sp. & 182,33 & 18,03 & 27,4 & 22,90 & 12,56 & 4580,2 & 18320,7 \\
A. salazarii & 21,96 & 19,47 & 43,00 & 2,11 & 9,61 & 1054,2 & 4216,7 \\
\hline
\end{tabular}

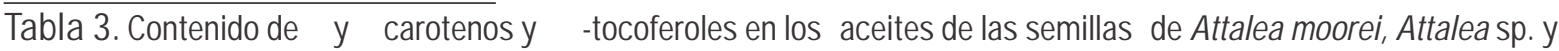
Attalea salazarii.

\begin{tabular}{|c|c|c|c|c|c|c|}
\hline \multirow[b]{2}{*}{ MUESTRA } & \multicolumn{3}{|c|}{ EN ACEITE } & \multicolumn{3}{|c|}{ EN SEM ILLA (CALCULADO) } \\
\hline & $\begin{array}{c}\alpha \text {-caroteno. } \\
\left(\text { ug g g }^{-1}\right)\end{array}$ & $\begin{array}{l}\beta \text { - caroteno. } \\
\text { (ug g-1) }\end{array}$ & $\begin{array}{c}\alpha \text { - tocoferol. } \\
\text { (mg L -1) }\end{array}$ & $\begin{array}{c}\alpha-\text { caroteno. } \\
(\mathrm{mg} / 100 \mathrm{~g}-)\end{array}$ & $\begin{array}{c}\beta \text { - caroteno. } \\
(\mathrm{mg} / 100 \mathrm{~g})\end{array}$ & $\begin{array}{c}\alpha \text {-tocoferol } \\
(\mathrm{mg} / 100 \mathrm{~g})\end{array}$ \\
\hline A. moorei & $795.22 \pm 0.42$ & $707.55 \pm 0.34$ & $7.7 \pm 0.05$ & 18.31 & 16.28 & 17.72 \\
\hline A. sp. & $2025.99 \pm 0.35$ & $3047.98 \pm 0.067$ & $7.5 \pm 0.07$ & 36.53 & 54.95 & 13.52 \\
\hline A. salazarii & $924.32 \pm 0.30$ & $758.10 \pm 0.03$ & $15.4 \pm 0.48$ & 17.99 & 14.76 & 29.98 \\
\hline
\end{tabular}

Tabla 4. Caracterización de hidrocarburos alifáticos, esteroles y alcoholes alifáticos de la fracción insaponificable de los aceites de las semillas de Attalea moorei, Attalea sp. y Attalea salazarii.
DETERMINACIÓN
A. moorei
A. sp.
A. salazarii

\begin{tabular}{lccc}
\hline Hidrocarburos alifáticos saturados (C10-C56), ppm & & & \\
\hline Hidrocarburos totales, ppm & 609,0 & 560,0 & 731,0 \\
Parafinas exógenas (aceite mineral) & 550,0 & 516,0 & 682,0 \\
\hline Determinación de Esteroles & & & \\
\hline Campesterol, \% & 17,5 & 14,3 & 17,72 \\
Estigmasterol, \% & 17,8 & 19,2 & 6,1 \\
Clerosterol, \% & 1,65 & 1,15 & -- \\
\hline -sitosterol, \% & 4,2 & 7,8 & 50,6 \\
Sitostanol, \% & 1,0 & 0,5 & 4,0 \\
\hline 5-Avenasterol, \% & 5,9 & 6,2 & \\
Esteroles totales, \% & 379,0 & 917,0 & 409,0 \\
\hline Alcoholes alifátic 0s & & \\
\hline C22-ol + C24-0l + C26-0l + C28-0l, ppm & 27,0 & 13,0 \\
\hline
\end{tabular}


Tabla 5. Valores del coeficiente de extinción específico a 25 ㅇ C de 232 y $270 \mathrm{~nm}$, correspondiente a los aceites de las semillas de Attalea moorei, Attalea sp. y Attalea salazarii.

\begin{tabular}{lcl}
\hline \multicolumn{1}{c}{ MUESTRA } & $\mathbf{K}_{\text {232nm }}$ & $\mathbf{K}_{\text {270nm }}$ \\
\hline Attalea salazarii & 0.272 & 0.175 \\
Attalea moorei & 0.283 & 0.409 \\
Attalea sp. $^{\prime}$ & 0.270 & 0.449 \\
Aceite de oliva comercial $^{1}$ & 3.04 & 0.195 \\
Aceite de oliva virgen $^{1}$ & 2.52 & 0.205 \\
Aceite de oliva refinado $^{1}$ & 1.85 & 0.375 \\
\hline
\end{tabular}

${ }^{1}$ Datos tomados de Paz Antolín et al.,(2000)

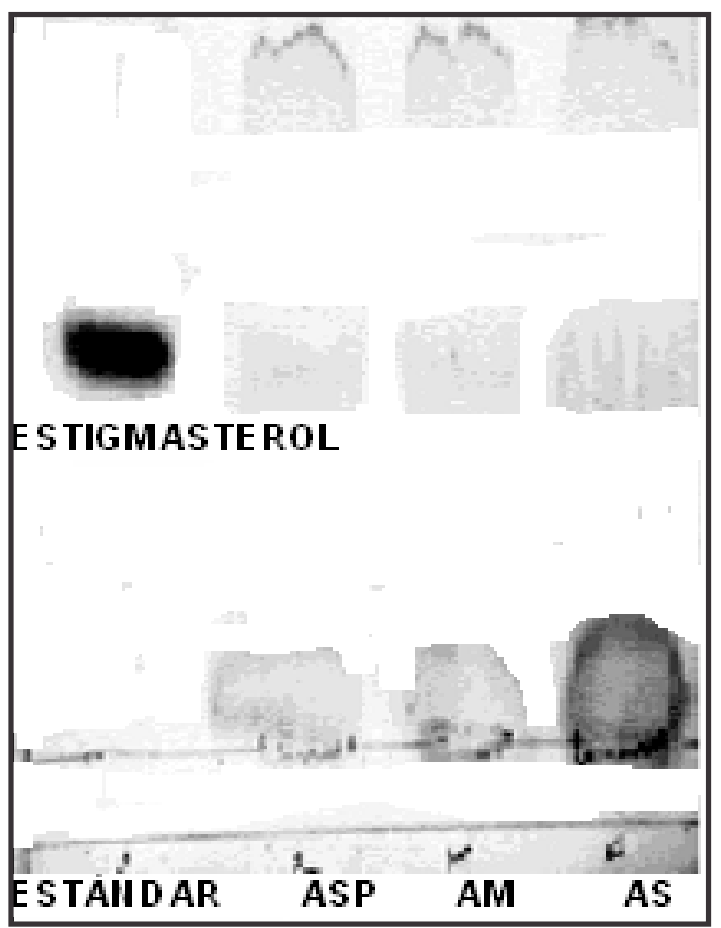

Figura 1. Estigmasterol en aceites de las semillas de Attalea moorei, Attalea sp. y Attalea salazarii, por cromatografía de capa fina (Punto de lado Izquierdo: estándar de estigmasterol y tres puntos siguientes: muestras) 
FO LIA

Amazónica

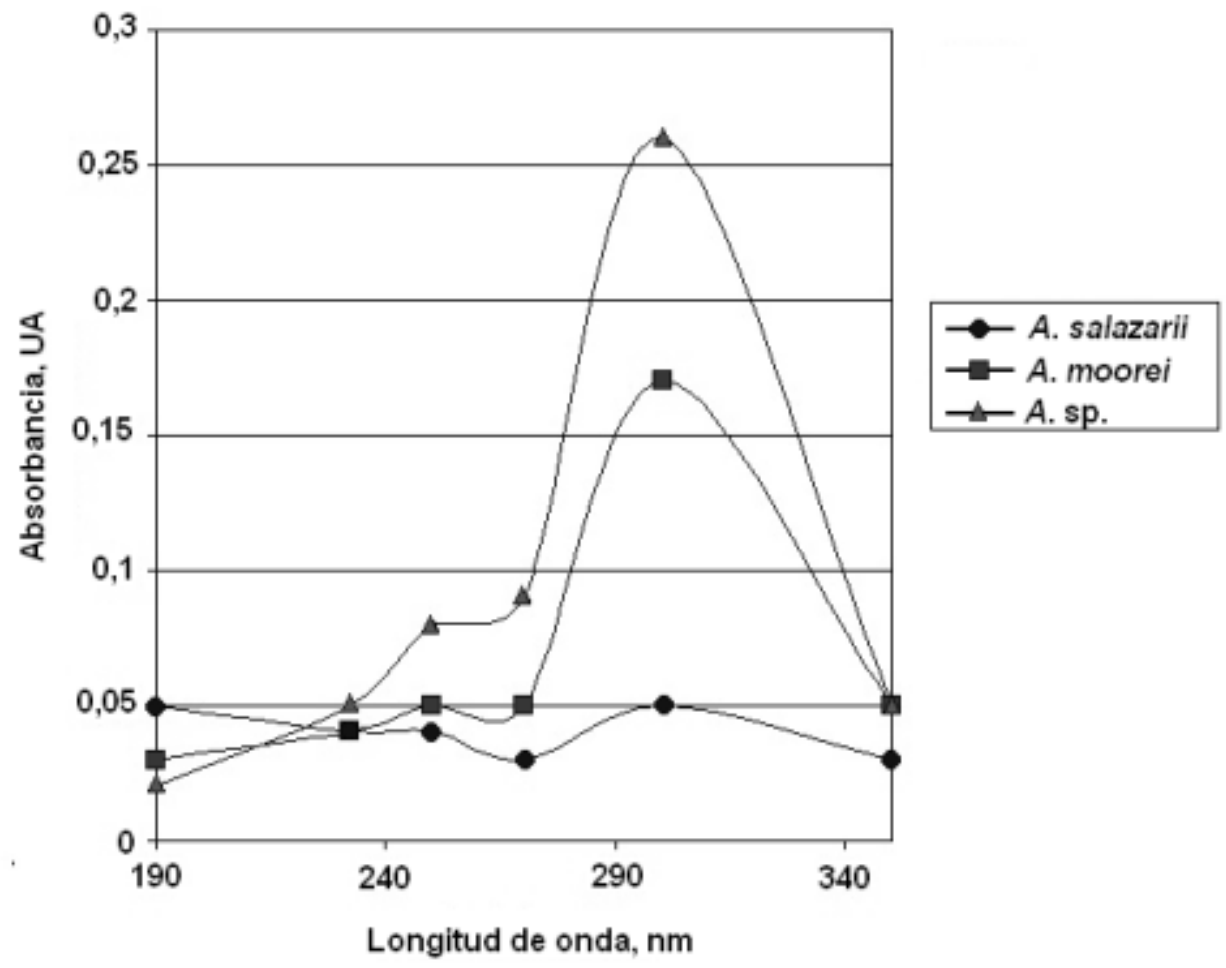

Figura 2. Espectros de absorción del aceite de las semillas de Attalea moorei, Attalea sp. y Attalea salazariii a 25 ○C en diferentes longitudes de onda.

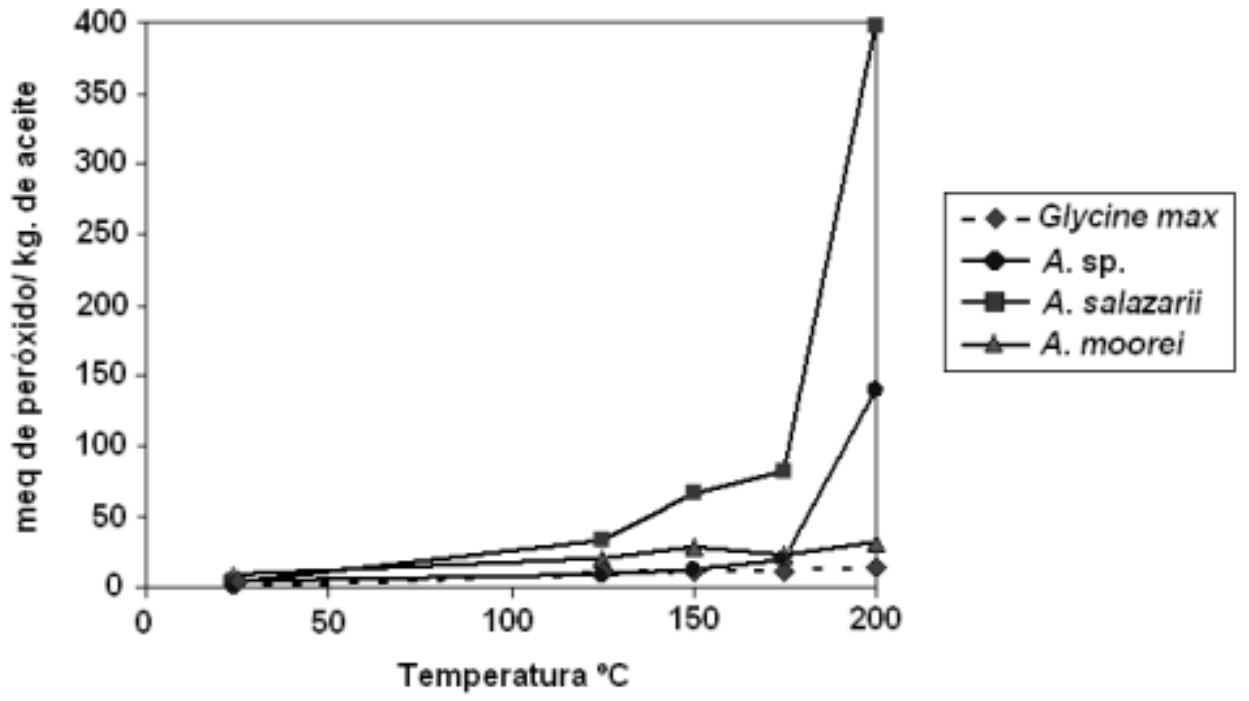

Figura 2. Cinética de oxidación del aceite de las semillas de Attalea moorei, Attalea sp. y Attalea salazarii. 


\section{CONCLUSIONES}

Se observa una importante concentración de carotenos, tocoferoles y esteroles en la fracción insaponifcable de los aceites de las especies del género Attalea, destacando la concentración de campesterol. $\beta$-sitosterol y estigmasterol.

Los aceites de Attalea salazarii y Attalea sp, son estables sin la adición de ningún antioxidante hasta la temperatura de $175^{\circ} \mathrm{C}$. y el de Attalea moorei hasta $200^{\circ} \mathrm{C}$. sin la adición de ningún antioxidante.

\section{AGRADECIMIENTO}

Al Programa de Ciencia y Tecnología - FINCyT por el apoyo financiero en la ejecución de este estudio.

\section{BIBLIOGRAFIA}

A.O.A.C. 2005. Official Methods of Analysis of AOAC International. Method 965.33. $18^{\circ}$ Edition. . Gaithersburg. Maryland - USA.

Arrubia, J.; Londoño, C.; Toro, A. 2008. Estudio de la Estabilidad Térmica del aceite de crisálida del Gusano de Seda Bómbix mori linn. Scientia et Técnica Año XIV. No 39. Universidad Tecnológica de Pereira. ISSN 0122-1701. pp. 410 -415 .

Awad. A.B.; Fink. C.S.; Williams. H.; Kim. U. 2001. In vitro and in vivo (SCID mice) effects of phytosterols on the growth and dissemination of human prostate cancer PC.-3 cells. European Journal of Cancer Prevention. 1(6): 507-513.

CEE 1991. Reglamento (CE) $\mathrm{N}^{\circ} 2568 / 91$ de la Comisión. del 11 de julio de 1991. relativo a las características de los aceites de oliva y de los aceites de orujo.

CEE 2003. Reglamento (CE) No 1989/2003. De la comisión de 6 de noviembre de 2003 por el que se modifica el Reglamento (CEE) $n^{\circ}$ 2568/91.Anexos V y XIX. Relativo a la característico de los aceites de oliva y de sus aceites de orujo de oliva y sobre sus métodos de análisis.

Chasquibol, N.; Lengua, L.; Delmás, D.; Bazán, D.; Aguirre, R.; Bravo, M. 2003. Alimentos funcionales. Clasificación e importancia. Rev. Per. Quim. Ing. Quim. 5(2):9-20.

Davila, E.; Merino, C.; Mejia, K.; Garcia, D.; Sauvain, M.; Sotero, V. 2011. Caracterización química de tres palmeras del género Attalea. Rev.Soc. Quím. Perú.77.(3): 218-224.
Febles, C.; Soto, C.; Saldaña, A.; García, B. 2002. Función de la vitamina E. Actualización. Rev. Cubana Estomatol. 40(1): 28-32

Ferreira, L. E. 2005. Manual das Palmeiras do Acre. Brasil. Instituto Nacional de Pesquisas / Universidade Federal do Acre. 30p.

Ghosh, T; Tapam, M.; Juapdesh, S. 2011. Evaluation of antitumor activity of stigmasterol a constituent isolated from Gracopa monniet Linn aerial part against. Ehrich Ascites carcinoma in mice. Oriental Pharmacy \& Experimental Medicine. 11(1):41-49.

Gomes da Silva, W.; Cortesi, N.; Rovellini, P. 1997. La nocciola brasiliana (Bertholletia excelsa H.B.K., famiglia delle Lecythidaceae). Nota II. I Lipidi-Studi sulla composizioni chimica. Rivi. Ital. Sostanze Grasse, Luglio, v. 74, p. 311-314

Jatunov, S.; Quesada, S.; Diaz, C.; Murillo, E. 2010. Carotenoid composition and antioxidant activity of the raw and boiled fruit mesocarp of six varieties of Bactris gasipaes. Archivos Latinoamericanos de Nutrición. 60 (1): 99-104.

Martinez, A. Carotenides. 2003. Facultad de Química Farmacéutica. Universidad de Antioquia. Colombia. 10p.

Nolasco, S.M.; Aguirrezabal, L.A.N.; Rapist, G.H. 2004. Tocopherol oil concentration in field grown sunflower is accounted for by oil weight per seed. J.Am.Oil Chem. Soc.. 81:1045-1051.

Paz, I.; Molero, M. 2000. Aplicación de la espectrofotometría UV - visible al estudio de la estabilidad térmica de aceites vegetales comestibles. Grasas y Aceites. 51(6): 424-428.

Rodríguez-Amaya, O. 1998. Carotenoides y preparación de alimentos: La retención de los carotenoides provitamina A en alimentos preparados, procesados y almacenados. UNICAMP. 105p.

Sayago, A.; Marin, M.J.; Aparicio, R.: Morales, M.J. 2007. Vitamina E y otros agentes vegetales. Grasas y Aceites. 58(1): 74-86.

Tano-Debrah, K.; Ohta, Y. 1996. Unsaponifiable matter constituents of crude shea fat extracted by different methods. J: Fac. Appl. Biol.Sci. Hiroshima Univ. 35: 163 -171.

Trevejo, E. 2003. Avances de la investigación en frutos oleaginosos de la amazonía peruana. UNAPCONCYTEC. $102 \mathrm{p}$.

Vasquez, P.; Freitas, L; Pavan, R.; Mancini filho, J.; 
Sotero, V. 2010. Chemical characterization and oxidative stability of the oils from three morphotypes of Mauritia flexuosa L.f., from peruvian amazon. Grasas y Aceites. 61(4):390397.

Villachica, H. 1996. Frutales y hortalizas promisorios de la amazonia. Tratado de Cooperación Amazónica. Secretaria Pro-Tempore. TCA. Lima
-Perú.p. 43-48.

Von Holtz, R.; Fuik, C.; Awad, A. 1998. $\beta$-sitosterol activites sphingomyelin cicle and induces apoptosis in LNCaP human prostate cells. Nutrition. 32(1): 8-12.

www.mobot.org/MOBOT/research/peru/diez_años_ perú.shtml, accesado ene 2009 